J. Indones. Math. Soc. (MIHMI)

Vol. 14, No. 2 (2008), pp. 111-119.

\title{
PRIMITIVE IDEALS OF TOEPLITZ ALGEBRA OF ORDERED GROUPS
}

\author{
RIZKY ROSJANUARDi
}

\begin{abstract}
The topology on primitive ideal space of Toeplitz algebras of totally ordered abelian groups can be identified through the upwards-looking topology if and only if the chain of order ideals is well-ordered. We describe the topology on primitive ideal space of Toeplitz algebra of totally ordered abelian groups when the chain of order ideals is not well ordered.
\end{abstract}

Received 4-6-2008, Accepted 6-12-2008.

2000 Mathematics Subject Classification: 46L55

Key words and Phrases: Toeplitz algebra, totally ordered group, primitive ideal.

Rizky Rosjanuardi: Department of Mathematics Education, Universitas Pendidikan Indonesia (UPI),

Bandung 40154, Indonesia.

E-mail: rizky@upi.edu. 